\title{
Krill Oil-Incorporated Liposomes As An Effective Nanovehicle To Ameliorate The Inflammatory Responses Of DSS-Induced Colitis
}

This article was published in the following Dove Press journal: International Journal of Nanomedicine

\author{
Jin-Hee $\mathrm{Kim}^{\mathrm{l}} *$ \\ Soon-Seok Hong ${ }^{1} *$ \\ Myoungsoo Lee ${ }^{2}$ \\ Eun-Hye Lee' \\ Inmoo Rhee' \\ Sun-Young Chang ${ }^{2}$ \\ Soo-Jeong Lim' \\ 'Department of Integrated Bioscience \\ and Biotechnology, Sejong University, \\ Seoul 05006, Republic of Korea; \\ ${ }^{2}$ Laboratory of Microbiology, College of \\ Pharmacy, and Research Institute of \\ Pharmaceutical Science and Technology \\ (RIPST), Ajou University, Suwon 16499 \\ Republic of Korea
}

*These authors contributed equally to this work
Correspondence: Sun-Young Chang Laboratory of Microbiology, College of Pharmacy, and Research Institute of Pharmaceutical Science and Technology (RIPST), Ajou University, Suwon 16499 , Republic of Korea

$\mathrm{Tel}+82312193454$

Fax +82312193435

Email sychang@ajou.ac.kr

Soo-Jeong Lim

Department of Integrated Bioscience and Biotechnology, Sejong University, 209

Neungdong-ro, Gwangjin-gu, Seoul 05006 , Republic of Korea

Tel +82 234083767

Fax +82 234084334

Email sjlim@sejong.ac.kr
Background: Phosphatidylcholine (PC) and Omega-3 fatty acid (Omega-3) are promising therapeutic molecules for treating inflammatory bowel disease (IBD).

Purpose: Based on the IBD therapeutic potential of nanoparticles, we herein sought to develop Omega-3-incorporated PC nanoparticles (liposomes) as an orally administrable vehicle for treating IBD.

Methods: Liposomes prepared with or without Omega-3 incorporation were compared in terms of colloidal stability and anitiinflammatory effects.

Results: The incorporation of free Omega-3 (alpha-linolenic acid, eicosapentaenoic acid or docosahexaenoic acid) into liposomes induced time-dependent membrane fusion, resulting in particle size increase from $\mathrm{nm}$ to $\mu \mathrm{m}$ during storage. In contrast, krill oil incorporation into liposomes (KO liposomes) did not induce the fusion and the particle size maintained $<250$ $\mathrm{nm}$ during storage. KO liposomes also maintained colloidal stability in simulated gastrointestinal conditions and exhibited a high capacity to entrap the IBD drug, budesonide (BDS). KO liposomes greatly suppressed the lipopolysaccharide-induced production of pro-inflammatory cytokines in cultured macrophages and completely restored inflammation-impaired membrane barrier function in an intestinal barrier model. In mice subjected to dextran sulfate sodium-induced colitis, oral administration of BDS-entrapped KO liposomes suppressed tumor necrosis factor- $\alpha$ production (by 84.1\%), interleukin- 6 production (by $35.3 \%$ ), and the systemic level of endotoxin (by 96.8\%), and slightly reduced the macroscopic signs of the disease.

Conclusion: Taken together, KO liposomes may have great potential as a nanovehicle for oral delivery of IBD drugs.

Keywords: inflammatory bowel disease, phosphatidylcholine, liposomes, krill oil, Omega-3 fatty acid

\section{Introduction}

Inflammatory bowel diseases (IBD) are chronic inflammatory conditions that affect the gastrointestinal (GI) tract. Ulcerative colitis (UC) and Crohn's disease, the two major types of IBD, share some common symptoms, such as bleeding, diarrhea, and weight loss. The precise etiology of IBD is not fully understood yet, but it appears to involve genetic and environmental factors. ${ }^{1}$ No curative treatment is available; most of the current therapies use the frequent administration of anti-inflammatory and/or immunosuppressive drugs with the goal of suppressing abnormal immune activation, achieving remission, and preventing relapse. ${ }^{2}$ These therapies, however, 
often lead to serious adverse effects and exhibit incomplete clinical efficacy. Thus, a major goal in IBD research is the development of new therapeutic approaches.

Accumulating evidence suggests that the intestinal mucosal barrier plays a central role in the pathogenesis of IBD. ${ }^{3}$ The major component of this barrier is the epithelial layer, the luminal surface of which is covered by mucus. Phosphatidylcholine (PC) binds to mucins of the colonic mucus to establish a protective surface that prevents potentially toxic antigens, including bacteria, from adhering and penetrating. $\mathrm{PC}$ is also integrated into the plasma membrane of enterocytes, and participates in various signaling pathways associated with inflammation. ${ }^{4}$ The level of PC in the colonic mucus is markedly reduced in UC patients, allowing colonic bacteria to penetrate through the mucosal barrier and trigger nonspecific but aggressive immune responses. ${ }^{2}$ Based on these findings, it has been hypothesized that the local supplementation of PC could help normalize the colonic mucus PC content, re-constitute the mucosal barrier, and suppress inflammation in UC. Indeed, PC supplementation studies in animal models and patients have yielded very encouraging results $^{4,5}$ and PC supplementation is currently considered to be a potential approach for treating IBD without substantial adverse effects. ${ }^{2}$

Omega-3 fatty acids (Omega-3) are long chain polyunsaturated fatty acids that range from 18 to 22 carbon atoms in chain length and present the first of many double bonds at the third carbon. Dietary supplement with Omega-3 provides multiple health benefits, including the inhibition of various aspects of inflammation. The mechanisms underlying these effects involve the incorporation of fatty acid into cell membrane and the decreased activation of pro-inflammatory transcription factors. Omega-3 intake has been shown to attenuate colonic damage and inflammation in animal models of IBD. ${ }^{6}$ Similarly, krill oil (KO), which is a marine-derived oil that is rich in Omega-3, was found to reduce intestinal inflammation in association with improved epithelial integrity. ${ }^{7,8}$ Collectively, these results strongly suggest the potential of Omega-3 in ameliorating the inflammatory responses of IBD. However, Omega-3 exhibits chemical instability due to its intrinsic susceptibility to oxidative degradation, potentially limiting its health benefits. ${ }^{9}$

For IBD therapy, researchers have sought to develop formulations that can increase the local drug concentration and residence time in the inflamed tissue, leading to enhanced therapeutic activity. Intestinal transit times are frequently accelerated in IBD patients as a consequence of diarrhea, but nanoparticle formulations have been recognized as an efficient means to prolong the colonic passage of drugs. ${ }^{10}$ The mucus layer tends to be increased in inflamed/ulcerated regions, allowing mucus-adherent nanoparticles to localize in these regions. ${ }^{11}$ Following this adherence, the increased permeability of the mucosa can allow nanoparticles to passively accumulate in the gaps between the cells; the nanoparticles may then be taken up by phagocytic cells that populate the inflamed region. ${ }^{11}$ Given these tendencies, nanoparticles are considered to be an ideal formulation for IBD therapy.

Liposomes formed of PC bilayers represent first-generation nanoparticle drug carriers that are already in clinical use. ${ }^{12}$ The liposome encapsulation of chemically unstable compounds, such as Omega-3, can offer a physical barrier that prevents the oxidative degradation of these compounds. ${ }^{9}$ This, together with the anti-inflammatory activity and intestinal barrier activity of PC, suggests that Omega-3-incorporated liposomes may be a promising nanovehicle for effective IBD therapy.

Fatty acids are amphipathic, and are thus rapidly incorporated into PC membranes. ${ }^{13}$ The monounsaturated fatty acid, oleic acid, was intercalated between PC molecules at up to $33 \mathrm{~mol} \%$ and oleic acid-incorporated membranes retained the physical stability of conventional liposomes. ${ }^{14}$ Furthermore, oleic acid or its 2hydroxyl derivative-incorporated liposomes demonstrated multiple benefits as drug carriers, such as improved colloidal stability and hydrophobic drug-loading capacity. ${ }^{14,15}$ The hydrocarbon tails of Omega-3, due to the presence of multiple double bonds in the cis configuration, are much more curved and flexible than those of monounsaturated fatty acid, suggesting that it may affect the liposome structure differently. ${ }^{16}$ With this regard, researchers studying the cellular membrane structure from a biophysical perspective reported that the insertion of docosahexaenoic acid (DHA) causes phase separation and fusion of membranes, leading to marked structural destabilization. ${ }^{17}$ Assuming that the effect of Omega-3 on the liposomal membrane may differ depending on the chemical structure and insertion content of Omega-3, we herein explored the impact of Omega-3 on the physical stability, the anti-inflammatory activity, and intestinal barrier activity of liposomes in order to develop an effective nanovehicle platform for IBD therapy. 


\section{Materials And Methods}

\section{Reagents And Materials}

1,2-distearoyl-sn-glycero-3-phosphocholine (DSPC), 1,2-dioleoyl-sn-glycero-3-phosphocholine (DOPC), and dioleoyltrimethylaminopropane (DOTAP) were purchased from Avanti Polar Lipid Inc. (Alabaster, AL, USA). Budesonide (BDS), alpha-linolenic acid (ALA, $\geq 98 \%$ purity), and eicosapentaenoic acid (EPA, $\geq 98 \%$ purity) were obtained from Cayman Chemical (Ann Arbor, MI, USA). DHA ( $\geq 98 \%$ purity), cholesterol (CHOL), $\alpha$-tocopherol, mucin (from porcine stomach), and lysozyme (from chicken egg white) were purchased from Sigma-Aldrich (St. Louis, MO, USA). Lipopolysaccharide (LPS) was purchased from Invivogen (San Diego, CA, USA). Dextran sulfate sodium (DSS) was obtained from MP Biomedicals (Solon, OH, USA). Krill oil (NKO ${ }^{\circledR}$; specific composition, $42 \% \mathrm{w} / \mathrm{w}$ phospholipids, $\geq 26.5$ $\mathrm{w} / \mathrm{w} \%$ total Omega- $3, \geq 8.5 \% \mathrm{w} / \mathrm{w}$ DHA, $\geq 14.5 \% \mathrm{w} / \mathrm{w}$ EPA, and $0.125 \pm 0.025 \mathrm{w} / \mathrm{w} \%$ astaxanthin) was kindly provided by Neptune Technologies \& Bioresources Inc. (Quebec, Canada).

\section{Cell Lines And Culture Conditions}

RAW 264.7 (murine macrophage) and CaCO-2 (human intestinal epithelial) cell lines were obtained from American Type Culture Collection (Manassas, VA, USA). Cells were cultured in DMEM (Welgene, Daegu, Korea) supplemented with $10 \%$ thermal-inactivated FBS and 100 units $/ \mathrm{mL}$ penicillin/streptomycin (Gibco, Grand Island, NY, USA). Cells were grown in incubators in a humid atmosphere of $95 \%$ air and $5 \% \mathrm{CO}_{2}$.

\section{Preparation Of Liposomes}

Liposomes were prepared by freeze-drying method as reported earlier. ${ }^{18}$ Lipid mixtures were dissolved in tertiary butyl alcohol. When the incorporation of Omega-3 and/or BDS was required, appropriate amounts of each were dissolved together with the lipid mixture. The mixtures were freeze dried (FDU-1200, EYELA, Tohoku, Japan). The obtained lipid cakes were hydrated with $5 \%$ dextrose dissolved in distilled water. The liposome dispersions were shortly vortexed and sonicated for $1 \mathrm{hr}$ at above the known phase-transition temperature of PC by using ultrasonic cleaning bath (3510-DTH Ultrasonic cleaner, Branson, Danbury, CT, USA). To obtain a liposomal dispersion with improved homogeneity, additional sonication for 7 mins was performed by using a cell disruptor (Bioruptor ${ }^{\circledR}$, UCD-200 T, Cosmo Bio, Tokyo, Japan) set at $250 \mathrm{~W}$. The prepared liposomes were stored at $4^{\circ} \mathrm{C}$ until use.

\section{Physicochemical Characterization Of Liposomes}

Particle sizes of dispersed liposomes were measured by the dynamic light scattering method using a fiber-optic particle analyzer (FPAR-1000, Otsuka Electronics, Japan). Prior to measurement, liposomes were appropriately diluted with $5 \%$ dextrose solution. System was used in the auto-measuring mode and the particle size analysis data were assessed using CONTIN program provided by the manufacturer. Zeta potentials, the electrical potential at the shear plane of the liposomal particles, were determined using zetasizer (Zetasizer Nano ZSP, Malvern Instruments, Northampton, MA). Prior to measurement, samples were diluted 50-fold with deionized water to reach the analytical measurement range. Default instrument settings and automatic analysis were used for all measurements. Each measurement was carried out in duplicate.

To perform negative-stain transmission electron microscopy, liposome samples were dropped on 200 mesh copper grid coated with carbon and negatively stained with $2 \%$ uranyl acetate for 1 min. Excess stain was removed, allowed to air-dry completely and dried samples were examined by using Tecnai G2 spirit (FEI Company, Hillsboro, OR, USA) operating at $120 \mathrm{keV}$.

With liposome samples diluted to $23 \mathrm{mM}$ of total lipid concentration, differential scanning calorimetry (DSC) was performed using a NanoDSC ${ }^{\circledR}$ (TA instrument, DE, USA) at the scan rate of $1{ }^{\circ} \mathrm{C} / \mathrm{min}$ and $35-65^{\circ} \mathrm{C}$ temperature range. Five percent dextrose solution was used as a reference. Data were analyzed using Nano Analyze software provided by manufacturer.

Storage stability of liposomes was assessed by monitoring changes in their sizes and morphology when incubated at room temperature over time. The GI stability of liposomes was evaluated by monitoring changes in their sizes when $50 \mu \mathrm{L}$ of liposomes were incubated with $950 \mu \mathrm{L}$ of phosphate-buffered saline ( $\mathrm{pH}$ 7.4), simulated intestinal fluid (SIF, $6.8 \mathrm{~g} \mathrm{KH}_{2} \mathrm{PO}_{4} / 1 \mathrm{~L}$ water, $\mathrm{pH} 7.5$, with $10 \mathrm{~g} / \mathrm{L}$ pancreatin), or simulated gastric fluid (SGF, $2 \mathrm{~g} \mathrm{NaCl} / 1 \mathrm{~L}$ water, $\mathrm{pH} 1.2$, with pepsin $3.2 \mathrm{~g} / \mathrm{L}) .{ }^{19}$

To evaluate the adhesive properties of the liposomes, their adsorption onto charged proteins was determined by using mucin and lysozyme as negatively and positively charged model proteins, respectively. ${ }^{20,21}$ Lysozyme or mucin solution $(4 \mathrm{mg} / \mathrm{mL}$ in distilled water) was incubated with liposome dispersion (1:1, v/v, $40 \mu$ mole lipid per $1 \mathrm{~mL})$ for $1 \mathrm{hr}$ at $37^{\circ} \mathrm{C}$. Then, non-adsorbed lysozymes were 
separated by dialysis using a Franz-type vertical diffusion system in which dialysis membrane (molecular weight cutoff $100 \mathrm{kDa}$ for lysozyme and $1000 \mathrm{kDa}$ for mucin) was placed between donor chamber and receptor chamber. After 4 hrs, samples containing non-adsorbed proteins were withdrawn from the receptor chamber and the protein concentration was quantified by the UV-Vis spectrophotometer (Beckman Coulter, CA, USA) at $280 \mathrm{~nm} .4 \mathrm{mg} / \mathrm{mL}$ of each protein solution pre-incubated with $5 \%$ dextrose (liposome-free) underwent the same procedure and the dialyzed amount was used as a $100 \%$ control of free/non-adsorbed proteins.

\section{Determination Of BDS Concentration Encapsulated In Liposomes}

Prepared liposome dispersions were immediately filtered through a $0.8-\mu \mathrm{m}$ syringe membrane filter to remove the non-encapsulated/precipitated BDS. The concentration of encapsulated BDS was then determined by HPLC analysis. ${ }^{22}$ Fifty microliter of BDS-entrapped liposome dispersion was freeze-dried, dissolved in $0.5 \mathrm{~mL}$ of methanol, and then centrifuged at 10,000 rpm for 10 mins. Twenty microliter of the supernatant BDS sample was injected into the Nanospace SI-2 HPLC system (Shiseido Co., Ltd, Tokyo, Japan) equipped a mobile phase delivery pump (SP 3201) and UV-visible detector (SP 3002). A Capcellpak C18 column (UG120, Shiseido, Tokyo, Japan) was used to perform the isocratic elution with a mobile phase consisting of methanol and distilled water $(72: 28, \mathrm{v} / \mathrm{v})$, the flow rate of $0.8 \mathrm{~mL} /$ min, and column temperature of $40^{\circ} \mathrm{C}$. The detection wavelength of BDS was $243 \mathrm{~nm}$.

\section{In Vitro Effect Of KO Liposomes On Epithelial Barrier Function}

Epithelial barrier integrity was assessed by measuring transepithelial electrical resistance (TEER) with an epithelial volt-ohm meter (EVOM; World Precision Instruments, Berlin, Germany). Decreased TEER is a significant indicator of impaired barrier function.

Caco-2 cells, an intestinal epithelial cell line widely used as a model to study intestinal barrier function in vitro, were placed at a density of $3.75 \times 10^{5}$ cells per well onto a $24-\mathrm{mm}$ polycarbonate transwell insert plates $(0.4 \mu \mathrm{m}$ pore size and a surface area of $4.67 \mathrm{~cm}^{2}$ (Corning, NY, USA)). The filter was placed in 6-well cell culture plates. The cell culture medium was changed every 3 days until the cells were fully differentiated (TEER value $>1200 \Omega \cdot \mathrm{cm}^{2}$ ). A steady-state of TEER was achieved after about 3 weeks, indicating that the barrier function model was established. RAW 264.7 macrophages were separately seeded at $8.5 \times 10^{5}$ cells/well into 6-well tissue culture plate. After 2 days, fresh, serumfree DMEM media containing $100 \mathrm{ng} / \mathrm{mL}$ of LPS were given to cells to mimic the inflammation. After 5-hr LPS stimulation, the culture supernatants were collected and then applied to fully differentiate Caco-2 monolayer ( $1 \mathrm{~mL}$ per well) to induce the disruption of the monolayer. When to test the effect of liposomes on the barrier function impaired by the inflammation, liposome dispersions or an equal volume of vehicle were given to Caco-2 monolayer cells right after supernatant stimulation. After an additional incubation for 3 hrs, the monolayer cells were rinsed with Hank's Balanced Salts Solution (HBSS; Welgene, Daegu, Korea) for 10 mins and then TEER was measured. Measurements were carried out at a constant temperature at three different locations within each well. Wells containing only culture medium were used as a blank control.

$\operatorname{TEER}\left(\Omega \cdot \mathrm{cm}^{2}\right)=($ measured TEER - control TEER $) \times$ effective membrane area of the cell culture well

\section{In Vitro Effect Of KO Liposomes On The Level Of Pro-Inflammatory Cytokines}

For IL-6 detection, RAW 264.7 cells were seeded at $2.5 \times 10^{5}$ cells/well into 24-well tissue culture plate and incubated overnight. And then, the cells were given fresh DMEM without FBS. To evaluate the anti-inflammatory effect of liposomes or BDS solution (in DMSO), appropriate amount of each was applied to the cells. At 2-hr post-incubation, fresh media were given again and the cells were stimulated with $100 \mathrm{ng} / \mathrm{mL}$ of LPS for $6 \mathrm{hrs}$. Concentration of IL- 6 in the culture supernatants was quantified by murine IL-6 Quantikine $^{\circledR}$ ELISA kit (R\&D Systems Inc., MN, USA) according to the manufacturer's instructions. For tumor necrosis factor-alpha (TNF- $\alpha$ ) detection, RAW 264.7 cells were seeded at $1 \times 10^{6}$ cells/well into 6 -well plate with or without BDS solution or liposomes. After 2-hr incubation, cells were harvested and washed with DMEM media. The cells were re-plated at $1 \times 10^{4}$ cells/well into 96 -well and stimulated with the indicated concentration of $100 \mathrm{ng} / \mathrm{mL}$ of LPS for 24 hrs. TNF- $\alpha$ in the supernatant was then quantified using ELISA kit, as detailed by the manufacturer (R\&D Systems Inc., MN, USA).

\section{Induction Of DSS-Mediated Colitis In Mice}

All experiments were approved by the Institutional Animal Care and Use Committee of Ajou University (IACUC 
approval number 2017-0021) and followed by the guidelines of AAALAC international and the Laboratory Animal Act of Korean Food and Drug Administration. Six-week-old female C57BL/6NCrljOri mice purchased from Orient Bio (Seongnam-Si, Korea) were housed at $24 \pm 1{ }^{\circ} \mathrm{C}$ with a 12 -hr light/dark cycle and food/water available ad libitum. Colitis was induced by administration of autoclaved drinking water containing 2\% DSS for 5 consecutive days. To evaluate the progression of colitis, a disease activity index (DAI) score was assessed during the duration of experiment. The DAI was determined by combining scores of weight loss compared to initial weight, stool consistency, and bleeding (divided by three). Scores were defined as follows: weight loss: 0 (no loss), 1 (1-5\%), 2 (5-10\%), 3 (10-20\%), and $4(>20 \%)$; stool consistency: 0 (normal), 2 (loose stool), and 4 (diarrhea); and bleeding: 0 (no bleeding), 2 (slight bleeding), 4 (gross bleeding).

\section{In Vivo Effects Of Liposomes On DSS- Induced Colitis}

Prior to the induction of colitis, mice were randomized into five groups: the negative control group (no DSS, $n=4$ ), the vehicle-treated DSS group (positive control, $n=6$ ), the KO liposome-treated DSS group (KO liposome group, $\mathrm{n}=6$ ), the BDS-treated DSS group (BDS group, $\mathrm{n}=8$ ), and the BDS-encapsulated KO liposome-treated DSS group (BDS(+) KO liposome group, $\mathrm{n}=8$ ). Free BDS was prepared by dispersing BDS at a concentration of $53 \mu \mathrm{g} / \mathrm{mL}$ in oil-in-water emulsion containing $0.5 \%$ soybean oil (Sigma-Aldrich, St. Louis, MO, USA) inner core stabilized by $1 \%$ carboxymethylcellulose (Sigma-Aldrich, St. Louis, MO, USA). KO liposomes and BDS(+) KO liposomes were prepared with the same composition of lipid: KO mixture in the presence of BDS (53 $\mu$ g per $40 \mu$ mole of lipid mixture). BDS was given to mice at a dose of 0.4 $\mathrm{mg} / \mathrm{kg}$ BDS and liposomes were given to mice at a dose corresponding to $300 \mu \mathrm{mole} / \mathrm{kg}$ as a total lipid mixture. Positive control group received vehicle solution instead of treatment. Each treatment was given to mice by oral gavage at the scheduled time points.

Body weight and DAI were checked daily to assess the severity of colitis. At day 9 after administration of DSS, serum and colon tissue samples were collected. Colon length and weight were measured to assess disease severity. The distal colons were washed with PBS and fixed in $4 \%$ formaldehyde at $4{ }^{\circ} \mathrm{C}$. The tissues were dehydrated by gradually soaking them in alcohol and xylene and then embedded in paraffin. The paraffin-embedded specimens were cut into 5- $\mu \mathrm{m}$ sections, stained with hematoxylin and eosin, and viewed with a digital light microscope DS-Fi2 (Nikon, Tokyo, Japan). Colon tissues were subjected to pathological scoring, which was performed in a blinded manner by a skilled pathologist. ${ }^{23}$

To assess the effect of liposomes on inflammation in vivo, cytokine (TNF- $\alpha$, IL- 6 and IFN- $\gamma$ ) level in serum collected from experimental groups was measured using murine inflammatory cytokine beads array kit (CBA kit, BD biosciences, USA) according to the manufacturer's instructions. Briefly, serum was diluted twofold with dilution buffer in CBA kit. Diluted samples were incubated with cytokine anti-beads at room temperature for $2 \mathrm{hrs}$. Then, CBA data were acquired on BD FACSARIA III (BD bioscience, San Jose, CA, USA) and analyzed using FCAP array software for quantifying cytokine level of samples.

Serum level of circulating endotoxin LPS was determined by Pierce LAL chromogenic endotoxin quantitation kit (Thermo scientific, USA). Briefly, pooled serum diluted 50-fold with endotoxin-free water was dispensed into microplate well. After 5-min incubation at $37^{\circ} \mathrm{C}$, limulus amebocyte lysate was added, followed by addition of chromogenic substrate for detecting LPS. LPS concentration was calculated by measurement of absorbance of samples and standards at $405 \mathrm{~nm}$ on a plate reader.

\section{Statistical Analysis}

Statistically significant differences for results obtained under different experimental conditions were determined using the two-tailed unpaired Student's $t$-test or one-way ANOVA, Bonferroni's Multiple Comparison Test.

\section{Results}

\section{Effect Of Omega-3 Incorporation On The Physicochemical Properties Of Liposomes} Liposomes with varying compositions were prepared as described in Table 1. ALA (three double bonds), EPA (five double bonds), or DHA (six double bonds) were incorporated in liposomes in which DOPC $(\mathrm{C} 18: 1)$ with $a \leq 0^{\circ} \mathrm{C}$ gel-to-liquid crystalline phase-transition temperature or DSPC (C18:0) with a $>50^{\circ} \mathrm{C}$ phase-transition temperature was used as the main "fluid" and "rigid" PC components, which enabled us to also examine the impact of PC fluidity in our system. Alpha-tocopherol was supplemented as a protectant to inhibit the oxidative degradation of Omega-3. 
Table I Lipid Composition Used To Prepare Various Liposome Formulations. The Indicated Amount Of Each Component Was Used To Produce I mL Of Liposomal Dispersions

\begin{tabular}{|c|c|c|c|c|c|}
\hline Formulation & DOPC $(\mu \mathrm{mol})$ & DSPC $(\mu \mathrm{mol})$ & CHOL $(\mu \mathrm{mol})$ & $\alpha$-Tocopherol $(\mu \mathrm{mol})$ & Omega-3 (mg) \\
\hline I & 35 & - & 3 & 2 & - \\
\hline 2 & - & 35 & 3 & 2 & - \\
\hline 3 & 35 & - & 3 & 2 & ALA (6) \\
\hline 4 & - & 35 & 3 & 2 & ALA (6) \\
\hline 5 & 35 & - & 3 & 2 & EPA (6) \\
\hline 6 & - & 35 & 3 & 2 & EPA (6) \\
\hline 7 & 35 & - & 3 & 2 & DHA (6) \\
\hline 8 & - & 35 & 3 & 2 & DHA (6) \\
\hline 9 & 35 & - & 3 & 2 & $\mathrm{KO}(6)$ \\
\hline 10 & - & 35 & 3 & 2 & $\mathrm{KO}(6)$ \\
\hline II & 35 & - & 3 & 2 & KO (I8) \\
\hline 12 & - & 35 & 3 & 2 & KO (I8) \\
\hline
\end{tabular}

Abbreviations: DSPC, I,2-distearoyl-sn-glycero-3-phosphocholine; DOPC, I,2-dioleoyl-sn-glycero-3-phosphocholine; CHOL, cholesterol; ALA, alpha-linolenic acid; EPA, eicosapentaenoic acid; DHA, docosahexaenoic acid; KO, krill oil.
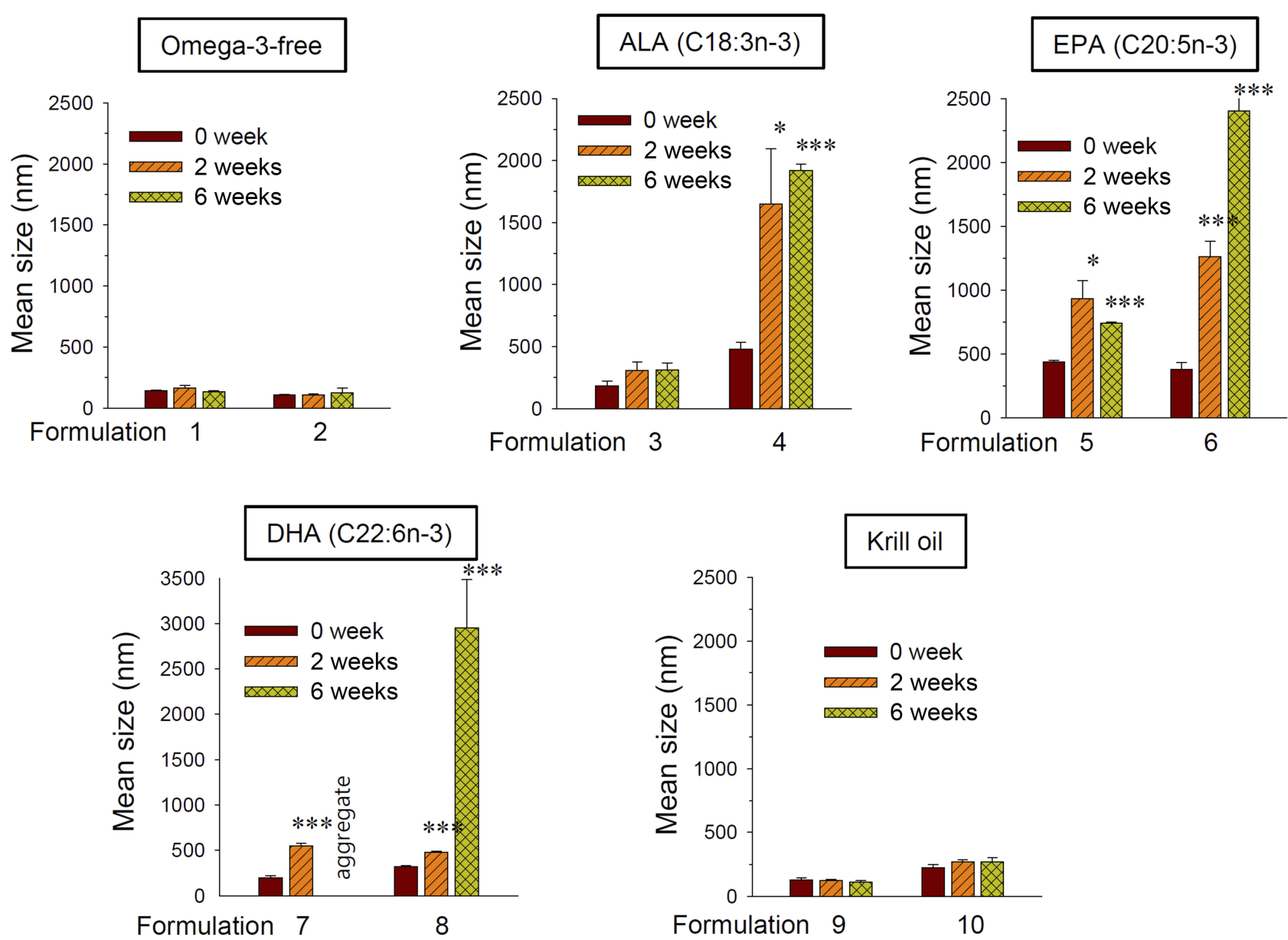

Figure I Effect of Omega-3 fatty acid incorporation on the time-dependent changes of mean particle size of liposomes.

Notes: Liposomes (Formulations I-10) were prepared from lipid mixtures as described in Table I. Prepared liposomes were incubated at room temperature for up to 6 weeks. Data are expressed as means $\pm S D(n=3 ; * p<0.01 ; * * *<0.001$, compared to the initial condition).

Abbreviations: ALA, alpha-linolenic acid; EPA, eicosapentaenoic acid; DHA, docosahexaenoic acid.

Omega-3-free lipid mixture produced very stable nanosized liposomes (Formulations 1 and 2) whose size remained unchanged up to 6-week incubation (Figure 1). Incorporation of ALA, EPA, or DHA (Formulations 3-8) 
produced larger vesicles that tended to grow in size over time: the mean sizes of DHA-, EPA-, and ALA-incorporated DSPC-based liposomes (Formulations 4, 6, and 8) increased by 9.3-, 6.3-, and 4.0-fold after 6-weeks, reaching $\geq 2 \mu \mathrm{m}$. DOPC-based liposomes (Formulations 3, 5, and 7) also tended to grow or aggregate in a time-dependent manner (Figure 1). In contrast, no significant size change was found during incubation of KO-incorporated liposomes (Formulations 9 and 10). Collectively, these data indicate the time-dependent size increases of liposomes incorporated with free Omega-3 (ALA, EPA, and DHA), but not those with KO.

To determine the highest $\mathrm{KO}$ content that could be stably incorporated into liposomes, we next prepared liposomes with varying contents of KO. After lyophilization of lipid mixture mixed with varying $\mathrm{KO}$ content (the first step to prepare liposomes), a lipid powder cake wet with $\mathrm{KO}$ was obtained with $\mathrm{KO}$ up to $\sim 27 \mathrm{mg}$ while the lipid mixture was found as dissolved in $\mathrm{KO}$ at $\geq 54 \mathrm{mg} \mathrm{KO}$. Consequently, a homogeneous dispersion of liposomes could be produced with a lipid mixture containing $\leq 27 \mathrm{mg} \mathrm{KO}$ (Figure 2A). Increasing the $\mathrm{KO}$ in the liposomes increased the negativity of the zeta potential of particles until a saturation point was reached at $27 \mathrm{mg} \mathrm{KO}$ (Figure 2B). KO-incorporated liposomes had a zeta potential lower than $-40 \mathrm{mV}$, indicating that $\mathrm{KO}$ incorporation yielded a partial electrostatic stabilization of the liposomes. Particle sizes of liposomes incorporated with 0-18 mg KO (Formulations 1, 9 and 11) did not significantly change during storage while those with $36 \mathrm{mg} \mathrm{KO}$ slightly increased at the final time point (Figure 2C). BDS,
(A)

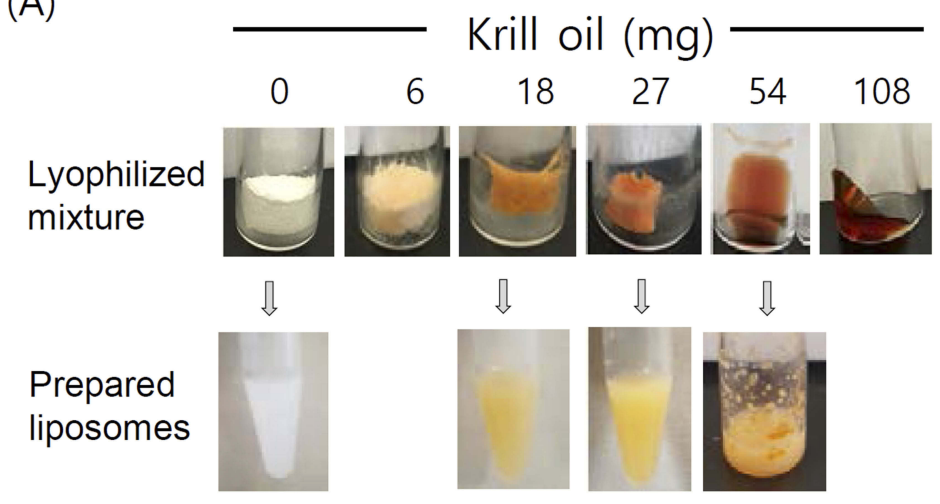

(C)

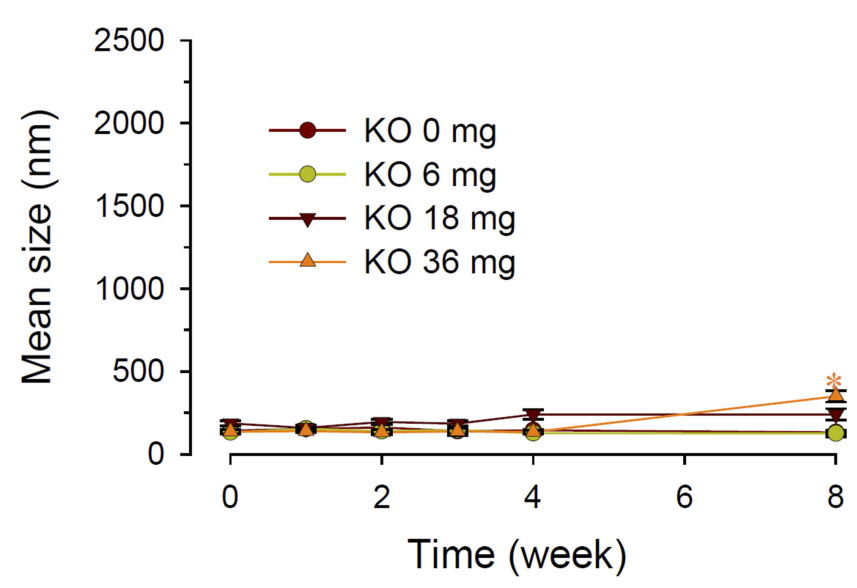

(B)

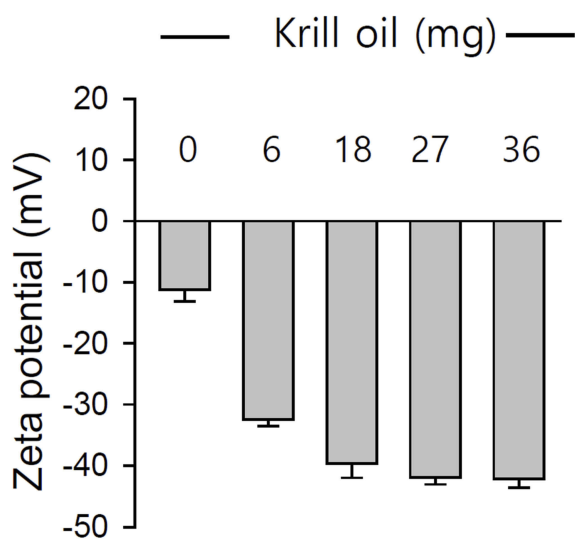

(D)

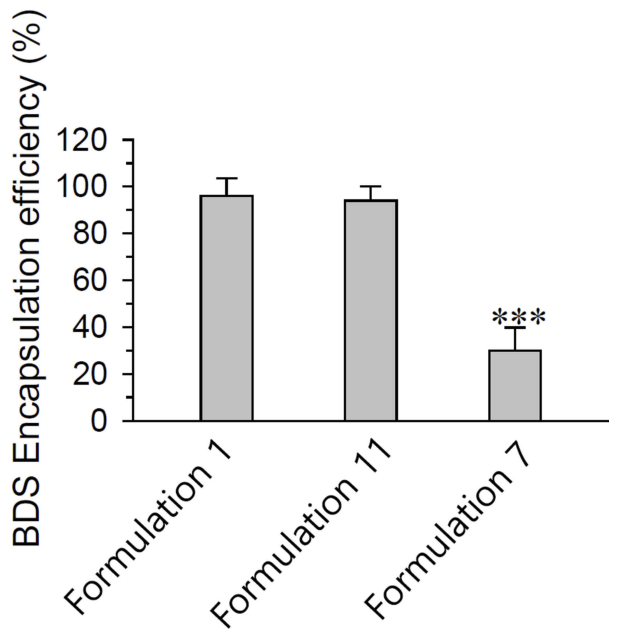

Figure 2 Effect of KO content on (A) physical state of lipid mixture obtained after lyophilization in the presence of varying amount of KO (top), and dispersion obtained from the corresponding lyophilized mixture (bottom), (B) zeta potential and (C) size changes of liposomes stored at room temperature. (D) Effect of Omega-3 incorporation on the liposomal encapsulation efficiency of BDS.

Notes: DOPC was used for the preparation of liposomes. A quantity of $40 \mu \mathrm{mol}$ of lipids were mixed with the indicated amounts of KO. For BDS encapsulation, I.5 mg BDS was dissolved together with the $40 \mu$ mole of lipid mixture prior to lyophilization. $* * * P<0.001$.

Abbreviations: DOPC, I,2-dioleoyl-sn-glycero-3-phosphocholine; KO, krill oil; BDS, budesonide; Omega-3, omega-3 fatty acid. 
an IBD drug, could be encapsulated with high efficiency (94-96\%) in both Omega-3-free (conventional) and $\mathrm{KO}$ liposomes (Formulations 1 and 11), but not in DHA-incorporated liposomes (Formulation 7) (Figure 2D). Together, our results show that KO-incorporated liposomes exhibited a high physical stability and drug-loading capacity, and $18 \mathrm{mg}$ KO per $40 \mu \mathrm{mol}$ lipids were maximal to exhibit these characteristics.

The time-dependent changes in liposome size and morphology were visualized by TEM. Liposomes incorporated with 6 or $18 \mathrm{mg} \mathrm{KO}$ (Formulations 10 and 12) were mostly spherical with dimensions of $\leq 150 \mathrm{~nm}$. In contrast, most of the EPA-incorporated liposomes (Formulation 6) were not smooth spheres, instead exhibiting edges (Figure 3A, top). After a 3-week incubation, the KO-incorporated liposomes retained their initial size and shape while the EPA liposomes were visualized as fused vesicles with diameters $>1 \mu \mathrm{m}$ (Figure 3A). Time-dependent fusion was also observed with the ALA- and DHA-incorporated liposomes (data not shown). Thus, the higher stability of KO-incorporated
(A)
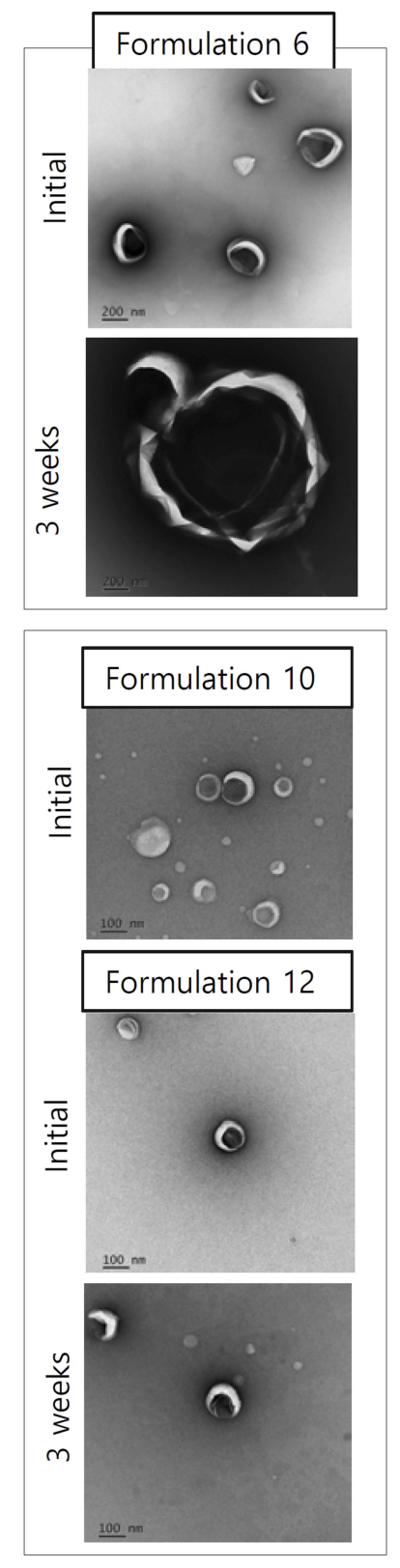

(B)

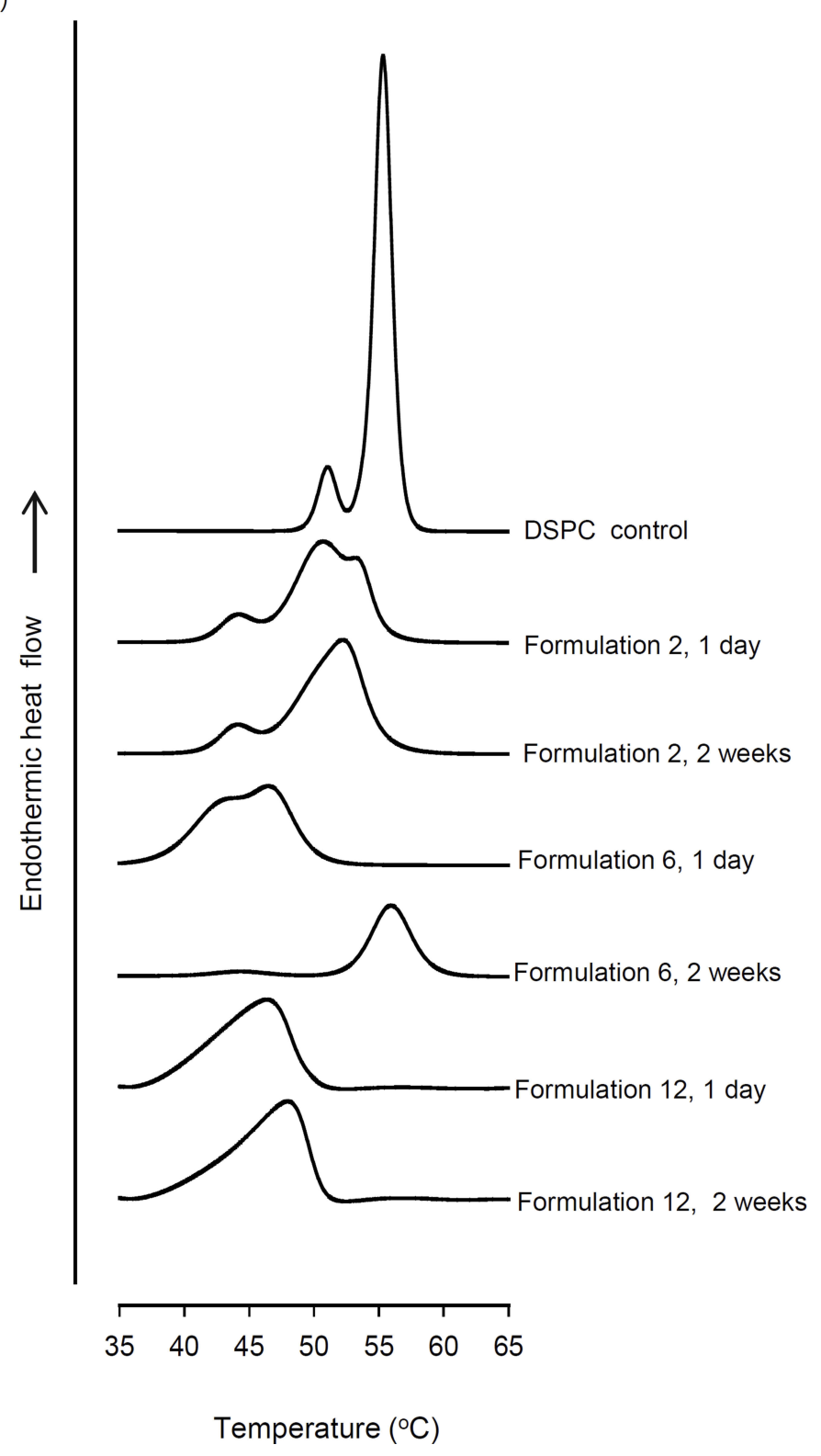

Figure 3 (A) TEM images and (B) phase-transition behavior of Omega-3-incorporated liposomes.

Notes: Formulations 2, 6, 10 and 12 were prepared. (A) Liposome samples taken at I day after preparation and after 3-week storage at room temperature were imaged at magnifications of $21,000 \times$ (Formulation 6) and 42,000× (Formulations 10 and 12). 
liposomes could reflect that these liposomes are more able to resist time-dependent fusion.

To further examine the mechanisms that might underlie the differences in liposomes incorporated with free Omega-3 and $\mathrm{KO}$, we performed DSC studies. DSPC alone showed pre- and major transition peaks at $51.0^{\circ} \mathrm{C}$ and $55.4^{\circ} \mathrm{C}$, respectively (Figure 3B), which was in good agreement with the literature. ${ }^{24}$ Previous studies showed that mixing $\mathrm{CHOL}$ and $\alpha$-tocopherol with rigid PC lowered the pre- and main transition temperatures and enthalpy, and reduced the packing order of gel-phase membranes, as shown in our Formulation 2. ${ }^{25}$ Here, we found that the incorporation of EPA (Formulation 6) significantly lowered and widened the major transition peak, which appeared split into two overlapping peaks, possibly indicating a phase separation in the PC membrane. In contrast, Formulation 12 showed a single phase-transition peak centering at $45.8^{\circ} \mathrm{C}$ without splitting (Figure 3B), suggesting that the lipid distribution in the $\mathrm{KO}-$ incorporated membrane was more homogeneous.

When Formulation 2 was incubated for 2 weeks, the pre- and major transition peaks of DSPC appeared at close to the original temperatures, although the major peak was somewhat sharper (Figure 3B). Marked changes were seen in the thermogram of Formulation 6 for the same duration: the original peak was abolished nearly to baseline and a new symmetrical peak appeared near a temperature of $56.0^{\circ} \mathrm{C}$, which appears to the major transition of DSPC. This suggests the extensive, time-dependent membrane rearrangement in EPA-incorporated liposomes, including the local formation of EPA-free DSPC membranes. Following storage, Formulation 12 did not exhibit any change in the phase-transition temperature range, except that the center of the major transition peak was slightly shifted to the right. Taken together, these data indicate that drastic, time-dependent PC membrane rearrangement is induced by EPA, but not by KO.

\section{In Vitro Anti-Inflammatory Effects Of KO Liposomes}

Formulation 11, renamed as KO liposomes from now on, was used for subsequent studies. When RAW264.7 macrophages were pretreated with KO liposomes prior to LPS stimulation, the productions of the proinflammatory cytokines, IL-6 and TNF- $\alpha$, were significantly decreased to an extent comparable to that seen in BDS-pretreated cells. Moreover, combined treatment with BDS and KO liposomes suppressed the levels of these cytokines even further (Figure 4A). Omega-3-free liposomes did not affect the production of proinflammatory cytokines (Figure 4A), suggesting that the potent anti-inflammatory effects of KO liposomes are mainly due to the incorporated $\mathrm{KO}$. Cell viability assay showed that $\mathrm{KO}$ liposome did not induce evident cell death in macrophages, excluding the possibility that the anti-inflammatory effects of $\mathrm{KO}$ liposomes are mediated by cytotoxicity (data not shown).

We next investigated the effect of our liposome formulations on the barrier function of $\mathrm{CaCo}-2$ cell monolayer. The application of the cell culture supernatant obtained from LPS-treated RAW264.7 cells reduced the TEER value of the monolayer to $39.9 \%$ of the control value (Figure 4B), suggesting that the membrane barrier was injured by components of the supernatant (eg, proinflammatory cytokines). When the impaired layer was post-treated with liposomes, the TEER value was recovered. Notably, $2.8 \mu \mathrm{L} / \mathrm{mL}$ of KO liposomes almost completely recovered the TER value $(\sim 98.0 \%)$, while the same volume of conventional liposomes yielded a recovery of only $\sim 64.7 \%$ (Figure $4 \mathrm{~B}$ ). Under the same conditions, 1 $\mu \mathrm{M}$ BDS did not affect the TEER value of the monolayer (data not shown). These findings indicate that supplementation with a liposomal vehicle, particularly one incorporating $\mathrm{KO}$, is very effective in restoring barrier function impaired by inflammatory factors.

\section{Physical Stability And Adsorption Properties Of KO Liposomes Under Simulated In Vivo Conditions}

Since the GI stability of a liposomal formulation is important for its ability to be administered orally, we assessed changes in the sizes of liposomes incubated for $24 \mathrm{hrs}$ with PBS, SGF, and SIF at $37^{\circ} \mathrm{C}$, to mimic the conditions in various regions of the GI tract. No size change was observed among the Omega-3-free liposomes, regardless of the incubation medium (Figure 5A). The KO liposomes maintained their initial size under all tested conditions, with the exception of incubation in SGF for $24 \mathrm{hrs}$. KO emulsions can be produced without the need for additional emulsifiers, given that Omega-3 is present in both its PCbound and free forms. ${ }^{26}$ Thus, we prepared $\mathrm{KO}$ emulsions and tested their GI stability for comparison. The particle size of $\mathrm{KO}$ emulsion started to increase after as little as $1 \mathrm{hr}$ of incubation in SGF (4.3-fold); after that time point, the size could not be determined due to extensive aggregation (Figure 5A). Considering that the gastric emptying 
(A)
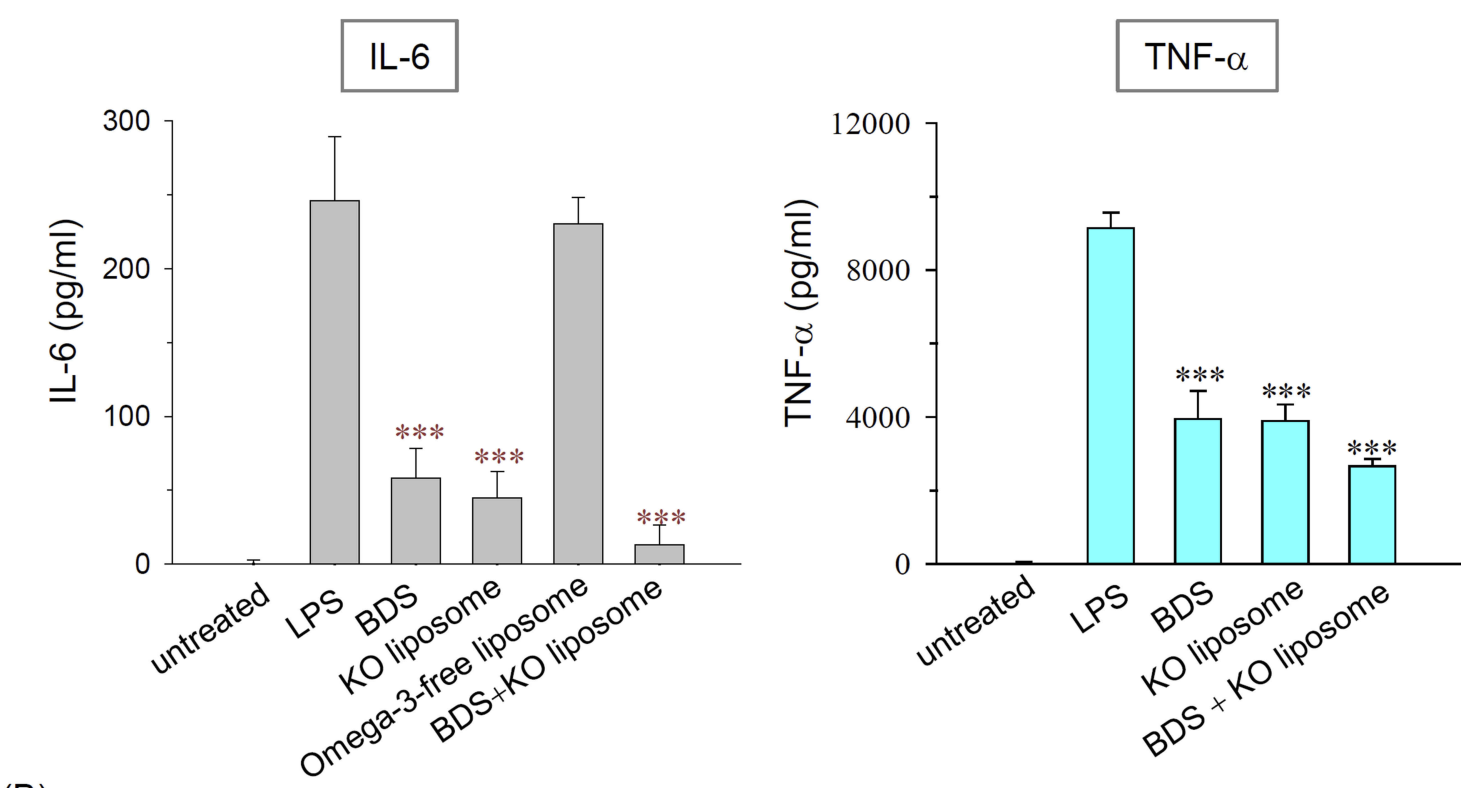

(B)

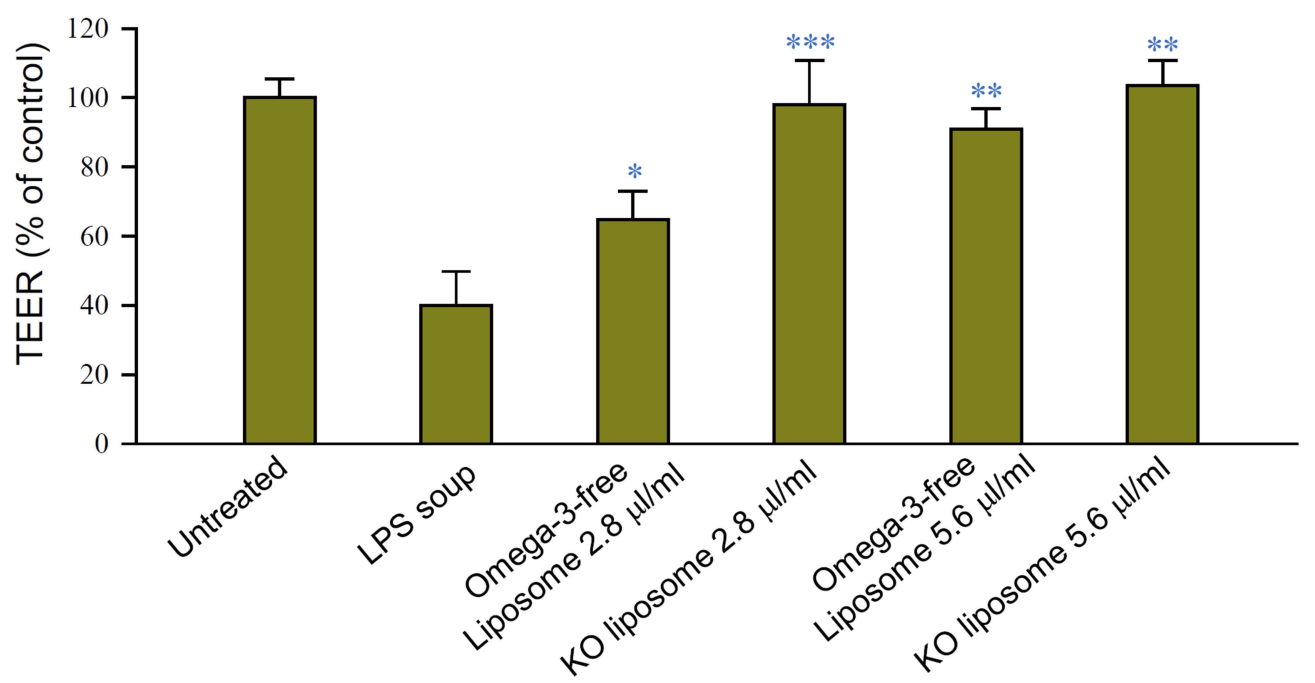

Figure 4 Effect of liposomes on (A) LPS-induced proinflammatory cytokine production in cultured macrophages and (B) the epithelial barrier function impaired by inflammatory cytokines.

Notes: (A) RAW 264.7 cells were pre-incubated with $250 \mathrm{nM}$ BDS and/or $5.6 \mu \mathrm{L} / \mathrm{mL}$ of Formulation I or Formulation II prior to LPS stimulation. (B) Barrier function was assessed by monitoring the TEER value of Caco- 2 cell monolayers, as described in the text. $N=3$; $* P<0.01$, $* * P<0.005$, and $* * * P<0.00$ I, compared to LPS group. Abbreviations: LPS, lipopolysaccharide; BDS, budesonide; TEER, transepithelial electrical resistance.

time is generally $3-4 \mathrm{hrs}$ after oral administration, ${ }^{27} \mathrm{R} 5$ these data indicate that $\mathrm{KO}$ liposomes may serve as an orally administrable therapeutic vehicle for treating IBD.

As anionic nanoparticles may exhibit stronger adherence to inflamed colon regions due to their strong electrostatic interaction with cationic proteins prevalent on cells commonly found in an inflamed region (eg, eosinophils), ${ }^{11}$ we investigated the protein adsorption properties of KO liposomes. As shown in Figure 5B, when KO liposomes were incubated with lysozyme as a cationic model protein, $\sim 93.5 \%$ of the lysozyme adsorbed to the liposomes. In contrast, we observed minimal adsorption of lysozyme to Omeg-3-free liposomes. Mucin, which is an anionic protein that coats the GI epithelium, exhibited higher protein adsorption on conventional liposomes compared to KO liposomes; however, the level of adsorption was below $10 \%$ in both cases whereas it was $>55 \%$ in case of DOTAP liposomes whose surface is highly positive (Figure 5B). ${ }^{28}$ These data suggest that the incorporation of KO into liposomes greatly increases their ability to adsorb cationic protein. 
(A)
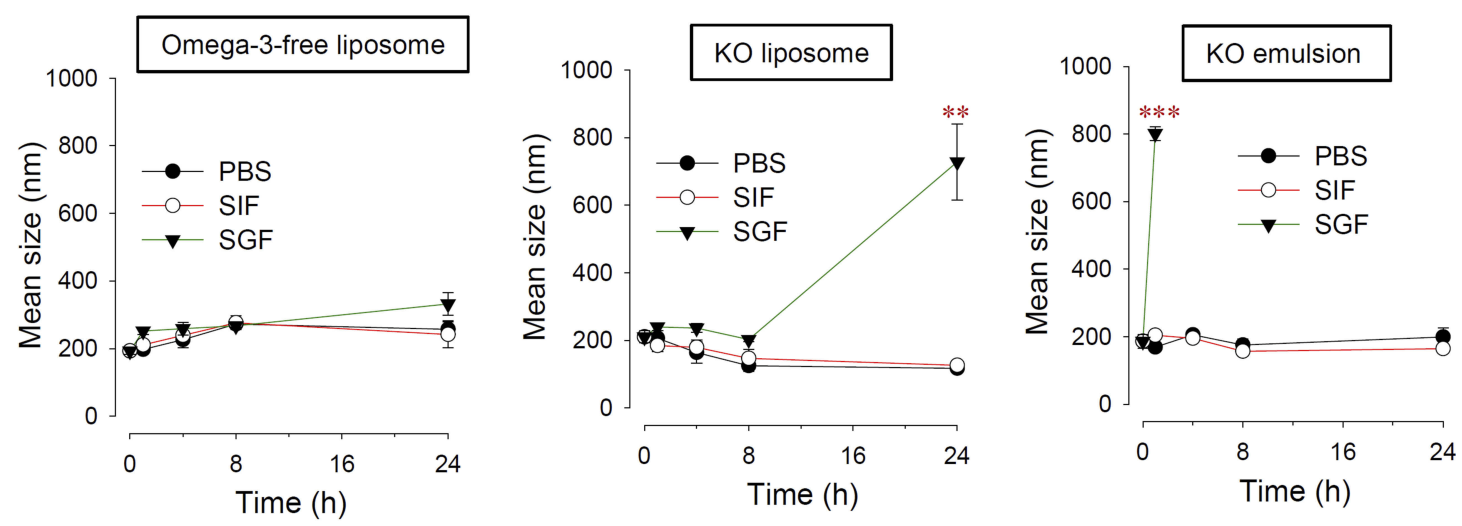

(B)
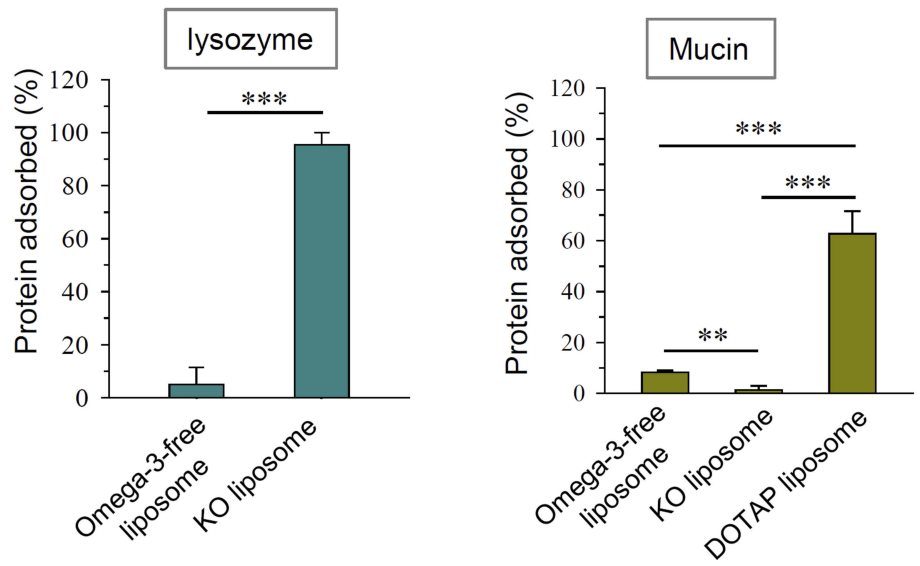

Figure 5 (A) Time-dependent size changes of Omeg-3-free liposomes, KO liposomes, and KO emulsion incubated with PBS, SGF, or SIF. (B) Adsorption of liposomes to charged proteins.

Notes: (A) KO emulsion was prepared by dispersing $18 \mathrm{mg} \mathrm{KO} \mathrm{in} \mathrm{I} \mathrm{mL} \mathrm{of} \mathrm{5 \%} \mathrm{dextrose} \mathrm{without} \mathrm{the} \mathrm{addition} \mathrm{of} \mathrm{any} \mathrm{additional} \mathrm{surfactant.} \mathrm{(B)} \mathrm{Liposomes} \mathrm{were} \mathrm{incubated}$ with lysozyme or mucin as described in the text. DOTAP liposomes were used as a positive control to confirm the adsorption of cationic liposome onto anionic proteins. $\mathrm{N}=4$; **P $<0.005 ; * * * P<0.00$ I, compared to the initial size

Abbreviations: SGF, simulated gastric fluid; SIF, simulated intestinal fluid; KO, krill oil; DOTAP, dioleoyltrimethylaminopropane.

\section{In Vivo Anti-Inflammatory Effects Of KO Liposomes}

Mice in the DSS group showed increased DAI compared with healthy controls, indicating that the DSS treatment successfully induced colitis. Treatment with BDS suspension, KO liposomes, or BDS-entrapped KO liposomes slightly decreased the DSS-induced level of DAI, although statistically not significant (Figure 6A). The colon length was shortened in DSS group, in accordance with the findings of other studies. ${ }^{29,30}$ BDS or KO liposome treatment slightly but significantly ameliorated DSS-induced colon shortening (Figure 6B). Pathological analysis of colon sections from DSS mice revealed signs of severe colitis, including crypt loss, submucosal edema, and infiltration of inflammatory cells. These parameters appeared to be reduced in the BDS-entrapped KO liposome group, but the pathologic scoring results did not significantly differ among the treatment groups (Figure 6C).

DSS administration significantly increased the serum levels of TNF- $\alpha$ and IL- 6 in mice, and tended to non-significantly increase the serum level of IFN- $\gamma$ (Figure 7A). BDS significantly decreased the DSS-induced production of TNF- $\alpha$ but increased that of IL-6. KO liposomes and $\mathrm{BDS}(+) \mathrm{KO}$ liposomes significantly decreased the serum levels of both TNF- $\alpha$ and IL-6 (Figure 7A), suggesting that orally administered KO liposomes exerted a potent antiinflammatory activity. 
(A)

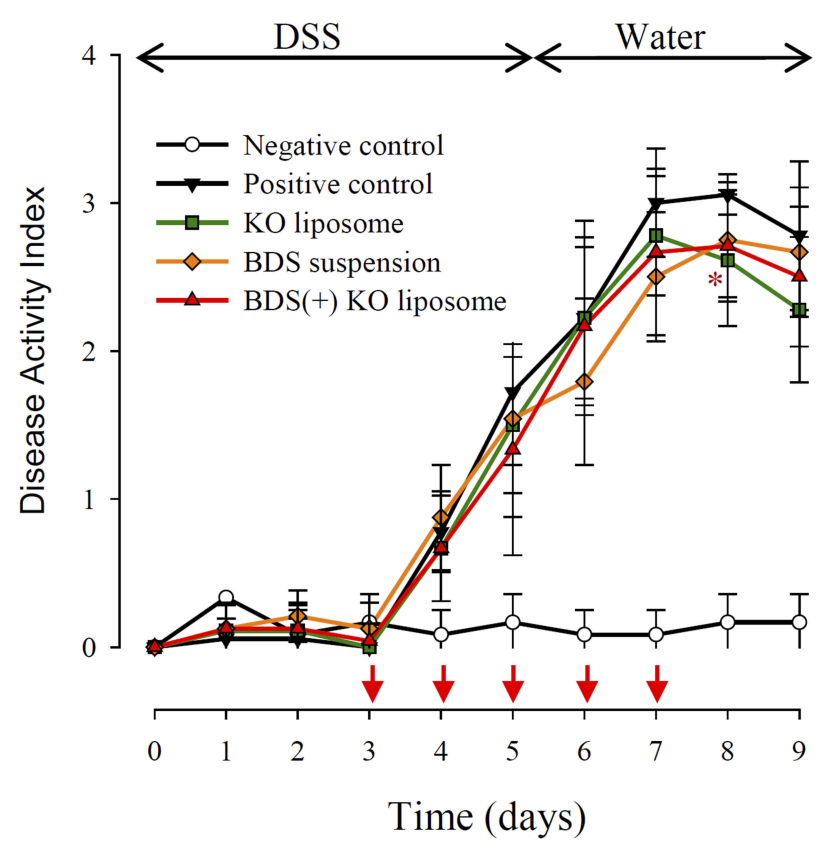

(B)

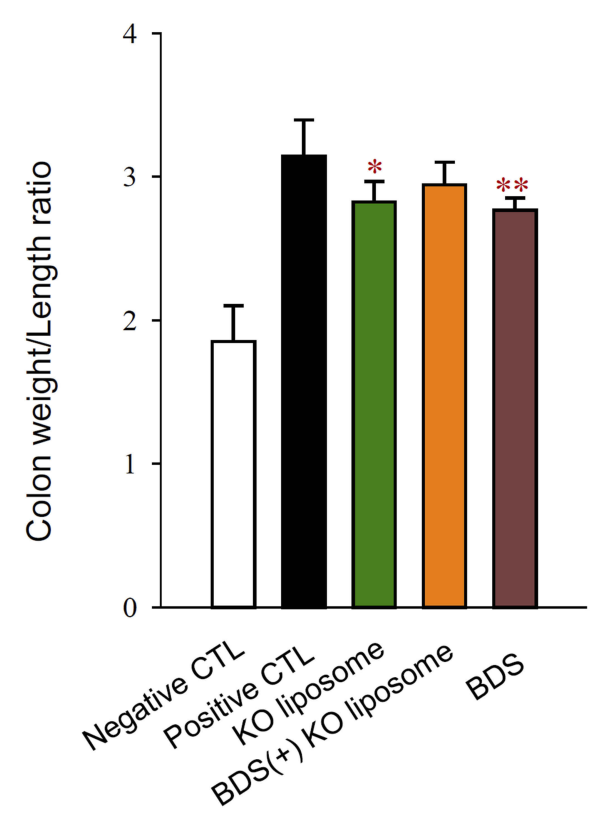

(C)

\begin{tabular}{|c|c|}
\hline Parameter & Grade \\
\hline Inflammation & $0-3$ \\
\hline Extent & $0-3$ \\
\hline Regeneration & $0-4$ \\
\hline Crypt damage & $0-4$ \\
\hline Percent involvement & $0-4$ \\
\hline
\end{tabular}

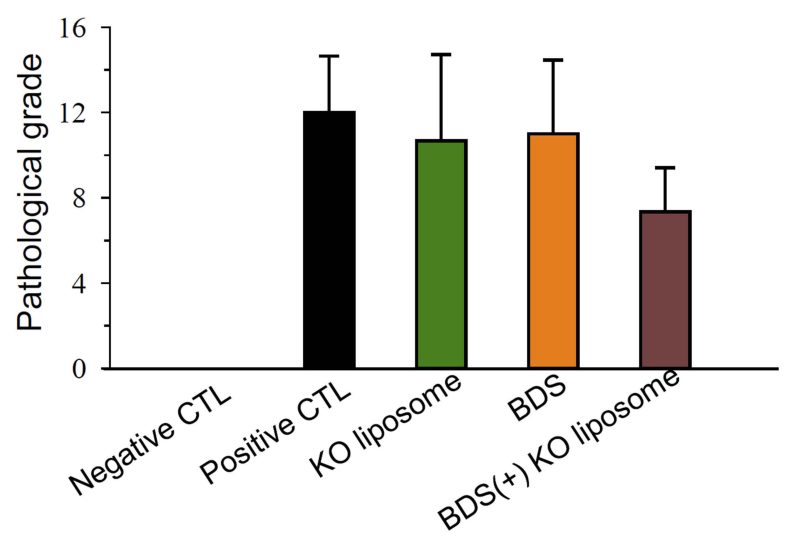

Negative CTL

Positive CTL

KO liposome

BDS

BDS(+) KO liposome

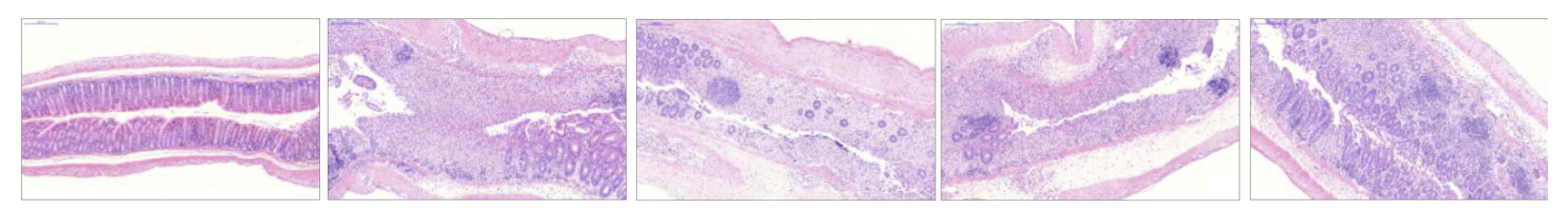

Figure 6 Effect of KO liposomes on macroscopic disease signs of DSS-induced colitis in mice.

Notes: (A) DAl change during the experimental period. Arrow $(\downarrow)$ indicates the treatment day. (B) Colon weight/length ratio determined on day 9 . (C) Representative H\&E images of distal colon and histological scoring of colonic inflammation for each group. Scale bar, $200 \mu$ m; ${ }^{*} p<0.05$, $* * p<0.005$ compared to positive control group. Abbreviations: DSS, dextran sulfate sodium; DAI, disease activity index; CTL, control; BDS, budesonide; KO, krill oil.

Serum endotoxin levels are known to be correlated with both the permeability of the intestinal mucosa and disease activity in IBD. ${ }^{31}$ As shown in Figure 7B, serum LPS was detectable in the positive control groups but not in the negative control groups, suggesting that LPS permeated through the impaired intestinal barrier in our DSS mouse model. KO liposomes, BDS(+) KO liposomes, or BDS suspension significantly reduced the serum level of LPS. Among them, BDS(+) KO liposomes exhibited the strongest decreasing effect: the serum LPS level was decreased by 3.3-, 31.2-, and 2.5-fold by KO liposomes, $\mathrm{BDS}(+) \mathrm{KO}$ liposomes, and BDS suspension, respectively. 

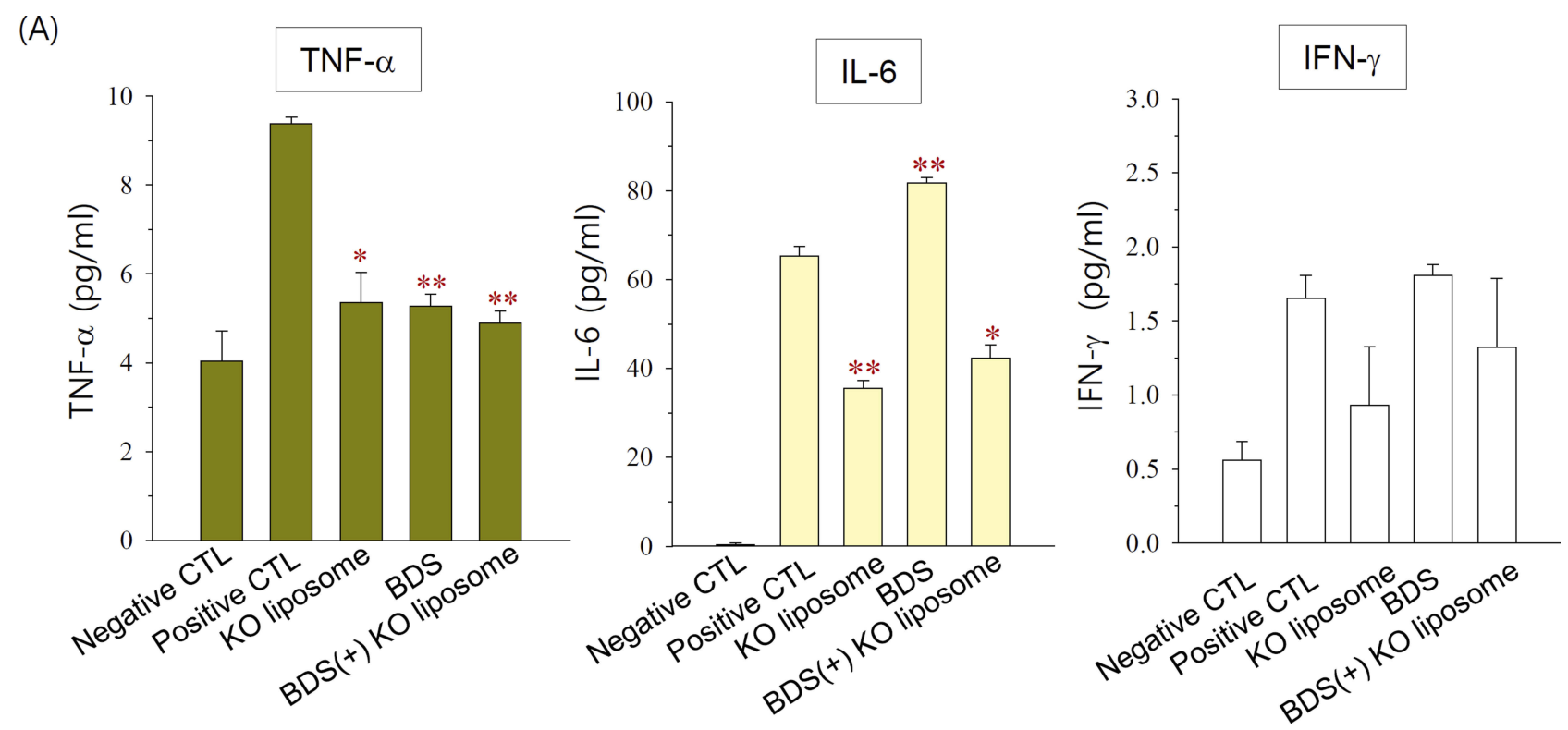

(B)

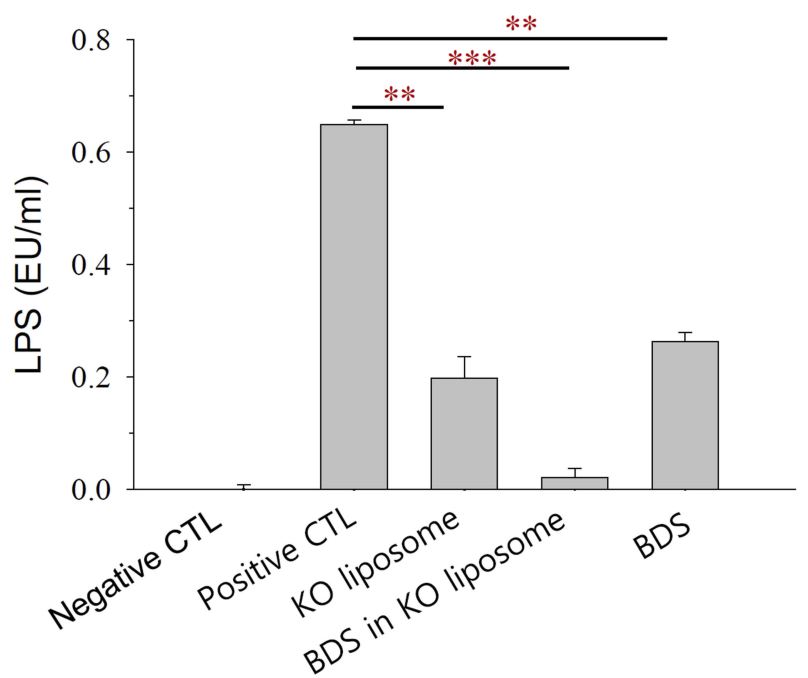

Figure 7 Anti-inflammatory effects of KO liposomes in DSS colitis mice, as assessed by the serum level of (A) pro-inflammatory cytokines and (B) LPS. Notes: Each point represents the mean $\pm S D(n=2) ; * P<0.05, * * P<0.005$, $* * * P<0.001$, compared to the positive control group.

Abbreviations: DSS, dextran sulfate sodium; CTL, control; BDS, budesonide; KO, krill oil; TNF- $\alpha$, tumor necrosis factor-alpha; IFN- $\gamma$, interferon-gamma; LPS, lipopolysaccharide.

These findings suggest that KO liposomes, particularly BDS-entrapped KO liposomes, can improve intestinal barrier function in a mouse model of colitis.

\section{Discussion}

As a first step in developing Omega-3-incorporated liposomes as an effective vehicle for IBD therapy, we set out to ensure that they exhibited physical stability before and after administration. However, we found that embedding free Omega-3 within the membrane induced the physical destabilization (fusion) of liposomes, as revealed by a drastic size increase (Figure 1 and Figure 3A) and alteration of the DSC thermogram (Figure 3B). Other groups had previously noted the physical instability of Omega-3-incorporated liposomes. ${ }^{17,32}$ Notably, Omega-3 has a non-cylindrical, inverted conical molecular shape reflecting that the diameter 
of the head group is much smaller than that of the hydrocarbon tail, and thus its incorporation between cylindrical PC molecules increases the built-in curvature stress of the membrane. ${ }^{33,34}$ This can cause marked structural changes of the lipid bilayer, and can be associated with phase separation and membrane fusion. ${ }^{32,35}$ Omega-3-induced edge formation may provide closer contact regions between membranes, thereby driving vesicle fusion (Figure $3 \mathrm{~A}){ }^{36}$ The more rapid destabilization of vesicles incorporating Omega-3 with higher numbers of cis double bonds (eg, DHA and EPA compared to ALA) may reflect the higher degree of curvature stress induced by the increased hydrocarbon tail chain bending of the fatty acids. It is not yet clear how KO liposomes retain a degree of stability comparable to that of Omeg-3-free liposomes despite having a high Omega-3 content. It is plausible that the incorporation of $\mathrm{KO}$ may cause less curvature stress due to the presence of Omega-3 mainly as phospholipid bound forms in KO. However, we found that the incorporation of docosahexaenoyl PC induced the time-dependent fusion of DSPC liposomes comparable to that of DHA (unpublished data), and these data suggest that the difference cannot be solely attributed to the different forms of Omega-3. KO has multiple components, including astaxanthin and vitamin $\mathrm{A}$, that may be fitted into spaces between Omega-3 and PC molecules to help lessen the curvature stress, and/or which could increase the electrostatic stabilization of particles by providing highly negative surface charges (Figure 2B).

In addition to being nanosized and electrostatically stable vehicles, KO liposomes exhibited high GI stability, high drug loading capacity, and strong adherence to cationic proteins, emphasizing their potential as an orally administrable IBD drug-carrying vehicle. These results indicate that using $\mathrm{KO}$ liposomes as an IBD drug carrier may yield increased drug accumulation in the inflamed intestinal region.

Our in vitro data demonstrate that $\mathrm{KO}$ liposomes themselves have potent anti-inflammatory effects. It seems likely that the liposome-incorporated $\mathrm{KO}$ blocked the production of pro-inflammatory cytokines by suppressing the LPS-induced expression of genes encoding inflammatory factors, such as

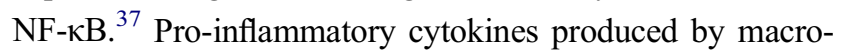
phages are known to act directly on intestinal epithelial cells to increase membrane permeability. ${ }^{38}$ Thus, the ability of KO liposomes to restore impaired barrier function may be associated with their suppressive effect on cytokine production. The administration of Omega-3-free liposomes or post-treatment with $\mathrm{KO}$ liposomes also induced recovery, suggesting that underlying mechanism involves the physical interaction of liposomes with the impaired barrier, presumably via the incorporation of PC into impaired membranes. The higher recovery effect of KO liposomes compared to Omeg-3-free liposomes seems likely to reflect both the supplementation of additional PC and the anti-inflammatory activity of KO.

The induction of colitis in DSS-treated mice was confirmed by monitoring increases in macroscopic (DAI, colon weight/length ratio, colonic inflammation) and biochemical (serum levels of TNF- $\alpha$, IL-6, IFN- $\gamma$, and LPS) parameters. Oral administration of $\mathrm{KO}$ liposomes greatly reduced the biochemical signs of colitis (Figure 7A and B) but only slightly reduced the macroscopic signs of the disease (Figure 6). Similarly, BDS, which is widely used to manage IBD, greatly reduced the biochemical parameters (serum levels of LPS and TNF- $\alpha$ ) but not the macroscopic signs of the disease under our experimental conditions. In this context, there are conflicting reports as to whether BDS improves the overall disease status in DSS-induced colitis, even though it shows anti-inflammatory activity in this model. $^{39,40}$ It is likely that certain experimental details, including the treatment schedule, may affect the effect of a treatment on the overall disease status.

The systemic levels of endotoxins (eg, LPS), which contribute to the inflammatory cascade in IBD patients, ${ }^{41}$ are positively correlated with clinical activity of $\mathrm{UC}^{31}$ Here, we show that KO liposomes can potently suppress the serum levels of LPS and proinflammatory cytokines, suggesting that the clinical activity of colitis is decreased in these mice. We speculate that the Omega-3 and PC provided by $\mathrm{KO}$ liposomes are incorporated into the impaired enterocyte membrane and/or bind to mucin and thereby re-establish the hydrophobic protective barrier in the inflamed/impaired region and decrease the permeation of LPS. BDS-entrapped KO liposomes suppressed LPS more than $\mathrm{KO}$ liposomes, further supporting the potential of KO liposomes as an effective drug carrier for IBD.

Additional studies, including efforts to reduce early intestinal absorption of the vesicles, are warranted to support the development of our KO liposomes as a clinically useful drug carrier for treating human IBD patients. It may be helpful to additionally coat the liposomes to allow timeor $\mathrm{pH}$-controlled drug release and support the accumulation of drugs beyond the duodenum. ${ }^{42}$ However, for such efforts, it will be necessary to address the potential variation of intestinal transit times and intraluminal $\mathrm{pH}$ depending on the patient's disease state. 


\section{Acknowledgments}

This work was supported by the National Research Foundation of Korea (NRF) and funded by the Ministry of Science, ICT and Future Planning (2015R1A2A2A01005783 and 2018R1A2B6003390 to S.J. Lim; 2017R1A2B4002419 to S.Y. Chang).

\section{Author Contributions}

All authors contributed to data analysis, drafting or revising the article, gave final approval of the version to be published, and agree to be accountable for all aspects of the work.

\section{Disclosure}

Jin-Hee Kim and Soo-Jeong Lim report a patent 10-20180079467 pending to Republic of Korea. The authors report no other conflicts of interest in this work.

\section{References}

1. Cohen LJ, Cho JH, Gevers D, Chu H. Genetic factors and the intestinal microbiome guide development of microbe-based therapies for inflammatory bowel diseases. Gastroenterology. 2019;156 (8):2174-2189. doi:10.1053/j.gastro.2019.03.017

2. Torres J, Danese S, Colombel JF. New therapeutic avenues in ulcerative colitis: thinking out of the box. Gut. 2013;62(11):1642-1652. doi:10.1136/gutjnl-2012-303959

3. Salim SY, Soderholm JD. Importance of disrupted intestinal barrier in inflammatory bowel diseases. Inflamm Bowel Dis. 2011;17(1):362381. doi:10.1002/ibd.21403

4. Stremmel W, Ehehalt R, Staffer S, et al. Mucosal protection by phosphatidylcholine. Dig Dis. 2012;30(Suppl 3):85-91. doi:10.1159/ 000342729

5. Karner M, Kocjan A, Stein J, et al. First multicenter study of modified release phosphatidylcholine "LT-02" in ulcerative colitis: a randomized, placebo-controlled trial in mesalazine-refractory courses. Am J Gastroenterol. 2014;109(7):1041-1051. doi:10.1038/ ajg.2014.104

6. Calder PC. Marine omega-3 fatty acids and inflammatory processes: effects, mechanisms and clinical relevance. Biochim Biophys Acta. 2015;1851(4):469-484. doi:10.1016/j.bbalip.2014.08.010

7. Costanzo M, Cesi V, Prete E, et al. Krill oil reduces intestinal inflammation by improving epithelial integrity and impairing adherent-invasive Escherichia coli pathogenicity. Dig Liver Dis. 2016;48 (1):34-42. doi:10.1016/j.dld.2015.09.012

8. Grimstad T, Bjorndal B, Cacabelos D, et al. Dietary supplementation of krill oil attenuates inflammation and oxidative stress in experimental ulcerative colitis in rats. Scand J Gastroenterol. 2012;47 (1):49-58. doi:10.3109/00365521.2011.634025

9. Wang Q, Lv S, Lu J, Jiang S, Lin L. Characterization, stability, and in vitro release evaluation of carboxymethyl chitosan coated liposomes containing fish oil. $J$ Food Sci. 2015;80(7):C1460-C1467. doi:10.1111/1750-3841.12929

10. Beloqui A, Coco R, Alhouayek M, et al. Budesonide-loaded nanostructured lipid carriers reduce inflammation in murine DSS-induced colitis. Int $J$ Pharm. 2013;454(2):775-783. doi:10.1016/j. ijpharm.2013.05.017

11. Collnot EM, Ali H, Lehr CM. Nano- and microparticulate drug carriers for targeting of the inflamed intestinal mucosa. $J$ Control Release. 2012;161(2):235-246. doi:10.1016/j.jconrel.2012.01.028
12. Choi YH, Han HK. Nanomedicines: current status and future perspectives in aspect of drug delivery and pharmacokinetics. $J$ Pharm Investig. 2018;48(1):43-60. doi:10.1007/s40005-017-0370-4

13. Leekumjorn S, Cho HJ, Wu Y, Wright NT, Sum AK, Chan C. The role of fatty acid unsaturation in minimizing biophysical changes on the structure and local effects of bilayer membranes. Biochim Biophys Acta. 2009;1788(7):1508-1516. doi:10.1016/j.bbamem.2009.04.002

14. Notman R, Noro MG, Anwar J. Interaction of oleic acid with dipalmitoylphosphatidylcholine (DPPC) bilayers simulated by molecular dynamics. $J$ Phys Chem B. 2007;111(44):12748-12755. doi:10.1021/jp0723564

15. Jang E-J, Choi WR, Kim S-Y, et al. 2-Hydroxyoleic acid-inserted liposomes as a multifunctional carrier of anticancer drugs. Drug Deliv. 2017;24(1):1587-1597. doi:10.1080/10717544.2017.1388452

16. Arouri A, Lauritsen KE, Nielsen HL, Mouritsen OG. Effect of fatty acids on the permeability barrier of model and biological membranes. Chem Phys Lipids. 2016;200:139-146. doi:10.1016/j.chemphyslip.2016.10.001

17. Ehringer W, Belcher D, Wassall SR, Stillwell W. A comparison of the effects of linolenic (18:3 omega 3) and docosahexaenoic (22:6 omega 3) acids on phospholipid bilayers. Chem Phys Lipids. 1990;54(2):79-88.

18. Hong SS, Kim SH, Lim SJ. Effects of triglycerides on the hydrophobic drug loading capacity of saturated phosphatidylcholine-based liposomes. Int $J$ Pharm. 2015;483(1-2):142-150. doi:10.1016/j. ijpharm.2015.02.013

19. Tapal A, Tiku PK. Complexation of curcumin with soy protein isolate and its implications on solubility and stability of curcumin. Food Chem. 2012;130(4):960-965. doi:10.1016/j.foodchem.2011.08.025

20. Jung IW, Han HK. Effective mucoadhesive liposomal delivery system for risedronate: preparation and in vitro/in vivo characterization. Int J Nanomedicine. 2014;9:2299-2306. doi:10.2147/IJN.S61181

21. Sciscione F, Pucci C, La Mesa C. Binding of a protein or a small polyelectrolyte onto synthetic vesicles. Langmuir. 2014;30(10):28102819. doi:10.1021/la500199w

22. Toropainen T, Velaga S, Heikkila T, et al. Preparation of budesonide/ gamma-cyclodextrin complexes in supercritical fluids with a novel SEDS method. J Pharm Sci. 2006;95(10):2235-2245. doi:10.1002/jps.20702

23. Dieleman LA, Palmen MJ, Akol H, et al. Chronic experimental colitis induced by dextran sulphate sodium (DSS) is characterized by Th1 and Th2 cytokines. Clin Exp Immunol. 1998;114(3):385-391. doi:10.1046/j.1365-2249.1998.00728.x

24. Sade A, Banerjee S, Severcan F. Concentration-dependent differing actions of the nonsteroidal anti-inflammatory drug, celecoxib, in distearoyl phosphatidylcholine multilamellar vesicles. $J$ Liposome Res. 2010;20(2):168-177. doi:10.3109/08982100903244492

25. Serro AP, Galante R, Kozica A, et al. Effect of tetracaine on DMPC and DMPC+cholesterol biomembrane models: liposomes and monolayers. Colloids Surf B Biointerfaces. 2014;116:63-71. doi:10.1016/j. colsurfb.2013.12.042

26. Wu Q, Uluata S, Cui L, et al. Physical and oxidation stability of selfemulsifying krill oil-in-water emulsions. Food Funct. 2016;7 (8):3590-3598. doi:10.1039/c6fo00045b

27. Minekus M, Alminger M, Alvito $P$, et al. A standardised static in vitro digestion method suitable for food - an international consensus. Food Funct. 2014;5(6):1113-1124. doi:10.1039/c3fo60702j

28. Kim SY, Lee SJ, Kim JK, Choi HG, Lim SJ. Optimization and physicochemical characterization of a cationic lipid-phosphatidylcholine mixed emulsion formulated as a highly efficient vehicle that facilitates adenoviral gene transfer. Int $J$ Nanomedicine. 2017;12:7323-7335. doi:10.2147/IJN.S146785

29. Kwon SH, Seo EB, Lee SH, et al. T cell-specific knockout of STAT3 ameliorates dextran sulfate sodium-induced colitis by reducing the inflammatory response. Immune Netw. 2018;18(4):e30. doi:10.4110/in.2018.18. e30

30. Yang JY, Kim MS, Kim E, et al. Enteric viruses ameliorate gut inflammation via toll-like receptor 3 and toll-like receptor 7-mediated interferon-beta production. Immunity. 2016;44(4):889-900. doi:10.1016/j. immuni.2016.03.009 
31. Gardiner KR, Halliday MI, Barclay GR, et al. Significance of systemic endotoxaemia in inflammatory bowel disease. Gut. 1995;36 (6):897-901. doi:10.1136/gut.36.6.897

32. Ibarguren M, Lopez DJ, Escriba PV. The effect of natural and synthetic fatty acids on membrane structure, microdomain organization, cellular functions and human health. Biochim Biophys Acta. 2014;1838(6):1518-1528. doi:10.1016/j.bbamem.2013.12.021

33. Arouri A, Mouritsen OG. Membrane-perturbing effect of fatty acids and lysolipids. Prog Lipid Res. 2013;52(1):130-140. doi:10.1016/j. plipres.2012.09.002

34. Mouritsen OG. Lipids, curvature, and nano-medicine. Eur J Lipid Sci Technol. 2011;113(10):1174-1187. doi:10.1002/ejlt.201100050

35. Onuki Y, Morishita M, Chiba Y, Tokiwa S, Takayama K. Docosahexaenoic acid and eicosapentaenoic acid induce changes in the physical properties of a lipid bilayer model membrane. Chem Pharm Bull (Tokyo). 2006;54(1):68-71. doi:10.1248/cpb.54.68

36. Malinin VS, Frederik P, Lentz BR. Osmotic and curvature stress affect PEG-induced fusion of lipid vesicles but not mixing of their lipids. Biophys J. 2002;82(4):2090-2100. doi:10.1016/S0006-3495(02)75556-2
37. Liu T, Zhang L, Joo D, Sun SC. NF-kappaB signaling in inflammation. Signal Transduct Target Ther. 2017;2. doi:10.1038/sigtrans.2017.23

38. Coskun M. Intestinal epithelium in inflammatory bowel disease. Front Med (Lausanne). 2014;1:24.

39. Naeem M, Choi M, Cao J, et al. Colon-targeted delivery of budesonide using dual $\mathrm{pH}-$ and time-dependent polymeric nanoparticles for colitis therapy. Drug Des Devel Ther. 2015;9:37893799. doi:10.2147/DDDT.S88672

40. Ocon B, Aranda CJ, Gamez-Belmonte R, et al. The glucocorticoid budesonide has protective and deleterious effects in experimental colitis in mice. Biochem Pharmacol. 2016;116:73-88. doi:10.1016/j. bcp.2016.07.010

41. Fukui $H$. Increased intestinal permeability and decreased barrier function: does it really influence the risk of inflammation? Inflamm Intest Dis. 2016;1(3):135-145. doi:10.1159/000447252

42. Patole VC, Pandit AP. Mesalamine-loaded alginate microspheres filled in enteric coated HPMC capsules for local treatment of ulcerative colitis: in vitro and in vivo characterization. $J$ Pharm Investig. 2018;48(3):257-267. doi:10.1007/s40005-017-0304-1
International Journal of Nanomedicine

\section{Publish your work in this journal}

The International Journal of Nanomedicine is an international, peerreviewed journal focusing on the application of nanotechnology in diagnostics, therapeutics, and drug delivery systems throughout the biomedical field. This journal is indexed on PubMed Central, MedLine, CAS, SciSearch ${ }^{\circledR}$, Current Contents ${ }^{\mathbb{B}} /$ Clinical Medicine,

\section{Dovepress}

Journal Citation Reports/Science Edition, EMBase, Scopus and the Elsevier Bibliographic databases. The manuscript management system is completely online and includes a very quick and fair peer-review system, which is all easy to use. Visit http://www.dovepress.com/ testimonials.php to read real quotes from published authors. 